Case Reports
in Dermatology

\title{
Development of Dermatitis Herpetiformis in Chronic Plaque Psoriasis
}

\author{
Ruby Lee ${ }^{a} \quad$ Yolanka Lobo $^{a} \quad$ Lynda Spelman $^{a, b}$ \\ aVeracity Clinical Research, Brisbane, QLD, Australia; ${ }^{b}$ Gabba Dermatology, \\ Brisbane, QLD, Australia
}

\section{Keywords}

Celiac disease · Dermatitis herpetiformis · Psoriasis

\begin{abstract}
Chronic plaque psoriasis is often associated with autoimmune bullous diseases. Dermatitis herpetiformis $(\mathrm{DH})$ is a rare immunobullous disease that has been linked to celiac disease (CD). To our knowledge, the coexistence of psoriasis and $\mathrm{DH}$ is uncommon, and has only been described in anecdotal reports. We report a case of chronic plaque psoriasis complicated by DH in a 60-year-old patient with no known history of CD or associated symptoms. In our patient, $\mathrm{DH}$ presented atypically as multiple vesicles along the edges of psoriatic plaques located on the back and hips, and as vesiculobullous eruptions on the fingers. The patient was successfully treated with a combination of dapsone and a gluten-free diet for $\mathrm{DH}$, and secukinumab for psoriasis. This case highlights the importance of screening for CD in patients with psoriasis, as well as other concomitant autoimmune diseases. A gluten-free diet should be trialled in psoriatic patients with positive CD serology.

\section{Introduction}

Chronic plaque psoriasis is frequently complicated by autoimmune bullous diseases such as pemphigoid and pemphigus [1]. To the best of our knowledge, the coexistence of psoriasis

\begin{tabular}{ll}
\hline & Ruby Lee \\
& Veracity Clinical Research \\
& Suite 18, Level 1/250 Ipswich Road, Woolloongabba \\
Brisbane, QLD 4102 (Australia) \\
ruby.cl.lee@gmail.com
\end{tabular}




\section{Case Reports in Dermatology}

Case Rep Dermatol 2021;13:141-147

DOI: $10.1159 / 000512870$

c) 2021 www.karger.com/cde

Lee et al.: Development of Dermatitis Herpetiformis in Psoriasis

and other immunobullous eruptions such as dermatitis herpetiformis (DH) is rare, and has only been described in anecdotal reports [2-4]. DH is an autoimmune bullous disease that has been linked to celiac disease (CD), a gluten-sensitive enteropathy. It is characterized by intensely pruritic papulovesicular eruptions classically seen on the extensor surfaces of the elbows, knees, buttocks and back. The literature suggests that patients with psoriasis have a higher risk of developing coexisting $\mathrm{CD}$ [5]. We hereby describe a case of chronic plaque psoriasis complicated by DH in a 60-year-old patient with no known history of CD or associated symptoms.

\section{Case Report}

A 60-year-old Caucasian man presented to our dermatology clinic with a 40-year history of chronic plaque psoriasis, worsening in severity over the last 5 years, and a 3-month history of intensely pruritic bullous eruptions on the back, hips and hands. His past medical and surgical history was significant for hypertension, type 2 diabetes mellitus, ischemic heart disease with prior double coronary artery bypass grafting and an L4-L5 lumbar spinal fusion. His medications included amlodipine, ramipril, atorvastatin, aspirin and metformin. Both the personal and the family history were negative for bullous and other cutaneous diseases. He had been treated unsuccessfully with multiple topical and systemic therapies for psoriasis, including betamethasone dipropionate, prednisolone and methotrexate.

On examination, he had multiple large, fixed, well-demarcated erythematous plaques on his back (Fig. 1) and typical features of nail psoriasis including onycholysis, pitting and splinter hemorrhages (Fig. 2). Of note, there were multiple vesicles present along the edges of the psoriatic plaques located on his back and hips (Fig. 3), as well as vesiculobullous eruptions on his fingers (Fig. 2). Histopathology of a vesicle taken from the plaque edge on the mid back showed subepidermal vesicle formation with dermal neutrophils and lower numbers of eosinophils, as can be seen in a number of settings including linear immunoglobulin A (IgA) bullous dermatosis and DH. No fungal organisms were seen with period acid-Schiff staining. Direct immunofluorescence of the perilesional skin on the back showed fine granular IgA deposits in the papillary dermis, without evidence of a linear pattern of IgA deposition within the basement membrane zone. These findings raised the possibility of DH and were to be correlated with clinical and laboratory findings.

The patient denied symptoms of CD such as altered bowel habits, abdominal pain, bloating and fatigue. CD serology showed anti-gliadin IgA titres of $69 \mathrm{U} / \mathrm{mL}(>20=$ positive), antigliadin IgG titres of $54 \mathrm{U} / \mathrm{mL}(>20=$ positive) and anti-tissue transglutaminase IgA titres of 31 $\mathrm{U} / \mathrm{mL}(>4=$ positive). Total serum IgA was $2.5 \mathrm{~g} / \mathrm{L}(0.7-3.6)$ and serum vitamin $\mathrm{D}$ was 70 $\mathrm{nmol} / \mathrm{L}(50-300)$. The patient was referred to a gastroenterologist for an endoscopy, which confirmed the diagnosis of CD and Barrett's esophagus with multifocal high-grade dysplasia. He subsequently underwent endoscopic mucosal resection of Barrett's segment and continues to be monitored via routine endoscopic surveillance.

A remarkable degree of improvement was seen in the patient's DH lesions following treatment with a gluten-free diet and dapsone at a daily dose of $25 \mathrm{mg}$. Anti-gliadin IgA decreased to $31 \mathrm{U} / \mathrm{mL}$ and anti-tissue transglutaminase IgA decreased to $6 \mathrm{U} / \mathrm{mL}$ after 10 months of treatment. The patient reported flares of DH within a day of gluten exposure. Dapsone was eventually tapered and ceased after 2 years owing to satisfactory management of $\mathrm{DH}$, with complete resolution of the vesicular lesions on the back and hips. Due to difficulty adhering to

\section{Karger'=}




\section{Case Reports in Dermatology}

Case Rep Dermatol 2021;13:141-147

DOI: $10.1159 / 000512870$

(c) 2021 www.karger.com/cde

Lee et al.: Development of Dermatitis Herpetiformis in Psoriasis

a gluten-free diet, he continues to experience intermittent flares of vesiculobullous eruptions on the hands. Despite improvement in DH, our patient's psoriasis remained severe, with a Psoriasis Area and Severity Index (PASI) score of 25.8. He was therefore commenced on secukinumab, a human monoclonal antibody that selectively targets interleukin (IL)-17A, at a dose of $300 \mathrm{mg}$ every month. Treatment with this agent over the past 8 years has resulted in significantly improved psoriatic skin lesions, with a PASI score of 1.8 on his most recent review.

\section{Discussion}

$\mathrm{DH}$ is a common extraintestinal manifestation of $\mathrm{CD}$, with a prevalence ratio of 1:8 [6]. To our knowledge, the coexistence of DH and chronic plaque psoriasis is rare and has only been described in case reports [2-4]. The current literature, however, supports an association between psoriasis and $\mathrm{CD}$, which would explain a possible connection between psoriasis and DH. A two-way meta-analysis by Acharya and Mathur [5] found that patients with psoriasis were 2.16-fold more likely to be diagnosed with $\mathrm{CD}$ and patients with $\mathrm{CD}$ were 1.8 -fold more likely to have psoriasis. A number of pathophysiologic mechanisms have been proposed. Immunologically, both $\mathrm{CD}$ and psoriasis are T cell-mediated disorders resulting from shared abnormalities in cytokine pathways. CD is the result of a T helper cell type (Th) 1 response in the small intestinal mucosa to dietary gluten. Th17 also has an important role in the pathogenesis of CD [7]. Similarly, psoriasis is a Th1-, Th17- and Th22-mediated skin disease that results in keratinocyte activation and proliferation. In addition, Zebrowska et al. [8], through immunohistochemical analysis of skin biopsies and measurement of serum concentrations, identified enhanced expression of IL-17 in patients with DH. This finding raises the possibility of using IL-17 targeted therapies in the treatment of DH. In our patient, the use of secukinumab, an IL17 inhibitor, resulted in significant improvement in psoriasis; however, its effect on DH is difficult to assess. Even though the patient reports an overall improvement and is no longer dependent on dapsone, he continues to experience recurrent flares of DH secondary to ongoing gluten exposure.

Woo et al. [9] observed that over $16 \%$ of patients with psoriasis have anti-gliadin IgA and IgG, anti-tissue transglutaminase IgA and anti-endomysial IgA antibodies. Furthermore, the authors discovered that a significantly higher portion of psoriatic patients with elevated CD antibodies had previously required or were currently on systemic immunosuppressants [9]. This implies that patients with psoriasis who demonstrate positive CD serology tend to have a more severe form of the disease, as was the case with our patient, who eventually required treatment with a biologic agent. Clinicians should consider prescription of biologic therapies sooner rather than later for the management of psoriasis particularly in this cohort of patients.

Michaëlsson et al. [10] demonstrated that psoriatic patients with positive anti-gliadin IgA or IgG had significant improvement in their PASI scores after 3 months of a gluten-free diet. The same group experienced flare-ups of psoriasis when gluten was reintroduced. Conversely, patients without antibodies to gliadin showed no improvement in their psoriasis following a gluten-free diet [10]. Clinicians should therefore consider screening for CD in all patients with psoriasis to ensure early diagnosis and management of $\mathrm{CD}$ and its complications. A gluten-free diet should be trialled in adults with psoriasis who have positive serologic markers of gluten sensitivity. Our patient had difficulty adhering to a gluten-free diet. Therefore, the improvement in psoriasis was limited and he eventually required treatment with a biologic agent.

\section{Karger'=}




\section{Case Reports in Dermatology}

\begin{tabular}{l|l}
\hline Case Rep Dermatol 2021;13:141-147 \\
\hline DOI: 10.1159/000512870 & $\begin{array}{l}\text { @ 2021 The Author(s). Published by S. Karger AG, Basel } \\
\text { www.karger.com/cde }\end{array}$ \\
\hline
\end{tabular}

Lee et al.: Development of Dermatitis Herpetiformis in Psoriasis

Positive CD serology should prompt immediate referral to a gastroenterologist. Patients with psoriasis who report bowel symptoms may also benefit from gastroenterology consultation.

Vitamin D deficiency can result from intestinal malabsorption in patients with CD. Studies suggest that vitamin D plays an important role in the maintenance of cutaneous barrier homeostasis in psoriasis $[11,12]$. Deficiency of vitamin D could hypothetically predispose susceptible individuals to psoriasis or worsen psoriasis severity. Our patient was commenced on vitamin D supplementation given that his level was on the lower end of the normal range, and also as a means to prevent worsening of disease severity in the setting of the known malabsorption of nutrients seen in patients with CD.

The atypical clinical presentation of DH in our patient, seen as multiple vesicles along the active edges of psoriatic plaques on the back and hips, suggests Koebnerization of the DH lesions as a result of the epidermal inflammation yielded by psoriasis. The Koebner phenomenon in DH has been reported in other cases, in which the authors describe the presence of DH lesions at sites of trauma due to tight-fitting undergarments [13, 14]. The potential for DH to demonstrate the Koebner phenomenon would explain its predilection for cutaneous eruptions on selective body areas such as the knees and elbows.

\section{Conclusion}

Psoriasis increases the likelihood of having other autoimmune diseases. This case highlights the importance of investigating for common coexisting conditions. Tissue biopsies should be performed on atypical lesions to ensure an accurate diagnosis. Clinicians should consider screening for $\mathrm{CD}$ in all psoriatic patients and trialling a gluten-free diet in those found to have positive CD serology.

\section{Acknowledgements}

The authors wish to thank our patient for allowing us to share his story.

\section{Statement of Ethics}

Written informed consent was obtained from the patient for publication of this case report and accompanying images in accordance with the World Medical Association Declaration of Helsinki.

\section{Conflict of Interest Statement}

The authors have no conflicts of interest to declare with respect to the research and/or authorship of this case report.

\section{Karger' $=$}




\section{Case Reports in Dermatology}

\begin{tabular}{l|l}
\hline Case Rep Dermatol 2021;13:141-147 \\
\hline DOI: 10.1159/000512870 & $\begin{array}{l}\text { ( ) 2021 The Author(s). Published by S. Karger AG, Basel } \\
\text { www.karger.com/cde }\end{array}$ \\
\hline
\end{tabular}

Lee et al.: Development of Dermatitis Herpetiformis in Psoriasis

\section{Funding Sources}

The authors received no financial support or sponsorship for the research and/or authorship of this case report.

\section{Author Contributions}

R. Lee: conception of the work, design of the work, acquisition and analysis of data, drafting the work, revising the work for important intellectual content, final approval of the version to be published, and agreement to be accountable for all aspects of the work in ensuring that questions related to the accuracy or integrity of any part of the work are appropriately investigated and resolved. Y. Lobo: design of the work, revising the work for important intellectual content, final approval of the version to be published, and agreement to be accountable for all aspects of the work in ensuring that questions related to the accuracy or integrity of any part of the work are appropriately investigated and resolved. L. Spelman: conception of the work, design of the work, acquisition and analysis of data, revising the work critically for important intellectual content, final approval of the version to be published, and agreement to be accountable for all aspects of the work in ensuring that questions related to the accuracy or integrity of any part of the work are appropriately investigated and resolved.

\section{References}

1 Dainichi T, Kabashima K. Interaction of Psoriasis and Bullous Diseases. Front Med (Lausanne). 2018 Aug;5:222.

2 Agusti-Mejias A, Messeguer F, García-Ruiz R, de Unamuno B, Pérez-Ferriols A, Sánchez-Carazo JL, et al. Concomitant dermatitis herpetiformis and plaque psoriasis: possible skin manifestations of celiac disease [in Spanish]. Actas Dermosifiliogr. 2011 Jul-Aug;102(6):471-3.

3 Gkalpakiotis S, Arenberger P, Sticova E, Sefrnova P, Arenbergerova M. Long-term combination therapy of ustekinumab and dapsone in a patient with psoriasis and dermatitis herpetiformis Duhring. J Dermatol. 2012 Dec;39(12):1074-5.

4 Aytekin S, Inalöz HS, Harman M, Akdeniz S, Deveci E, Inalöz SS. Coexistence of psoriasis vulgaris and bullous disorders. J Eur Acad Dermatol Venereol. 1999 Mar;12(2):185-7.

5 Acharya P, Mathur M. Association between psoriasis and celiac disease: a systematic review and metaanalysis. J Am Acad Dermatol. 2020 Jun;82(6):1376-85.

6 Reunala T, Salmi TT, Hervonen K, Kaukinen K, Collin P. Dermatitis Herpetiformis: A Common Extraintestinal Manifestation of Coeliac Disease. Nutrients. 2018 May;10(5):602.

7 Mazzarella G. Effector and suppressor T cells in celiac disease. World J Gastroenterol. 2015 Jun;21(24):7349-56.

8 Zebrowska A, Wagrowska-Danilewicz M, Danilewicz M, Stasikowska-Kanicka O, Cynkier A, SysaJedrzejowska A, et al. IL-17 expression in dermatitis herpetiformis and bullous pemphigoid. Mediators Inflamm. 2013;2013:967987.

9 Woo WK, McMillan SA, Watson RG, McCluggage WG, Sloan JM, McMillan JC. Coeliac disease-associated antibodies correlate with psoriasis activity. Br J Dermatol. 2004 Oct;151(4):891-4.

10 Michaëlsson G, Gerdén B, Hagforsen E, Nilsson B, Pihl-Lundin I, Kraaz W, et al. Psoriasis patients with antibodies to gliadin can be improved by a gluten-free diet. Br J Dermatol. 2000 Jan;142(1):44-51.

11 Barrea L, Savanelli MC, Di Somma C, Napolitano M, Megna M, Colao A, et al. Vitamin D and its role in psoriasis: an overview of the dermatologist and nutritionist. Rev Endocr Metab Disord. 2017 Jun;18(2):195205.

12 Umar M, Sastry KS, Al Ali F, Al-Khulaifi M, Wang E, Chouchane AI. Vitamin D and the Pathophysiology of Inflammatory Skin Diseases. Skin Pharmacol Physiol. 2018;31(2):74-86.

13 Campbell-D'Hue G, Estes SA. A Koebner phenomenon in dermatitis herpetiformis. J Am Acad Dermatol. 1983 Nov;9(5):767-8.

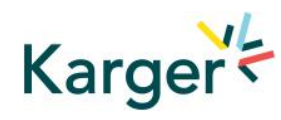




\section{Case Reports in Dermatology}

\begin{tabular}{l|l}
\hline Case Rep Dermatol 2021;13:141-147 \\
\hline DOI: 10.1159/000512870 & $\begin{array}{l}\text { C } 2021 \text { The Author(s). Published by S. Karger AG, Basel } \\
\text { www.karger.com/cde }\end{array}$ \\
\hline
\end{tabular}

Lee et al.: Development of Dermatitis Herpetiformis in Psoriasis

14 Bonsmann G, Hamm H. The Köbner phenomenon in Duhring dermatitis herpetiformis [in German]. Hautarzt. 1993 Jan;44(1):30-3.

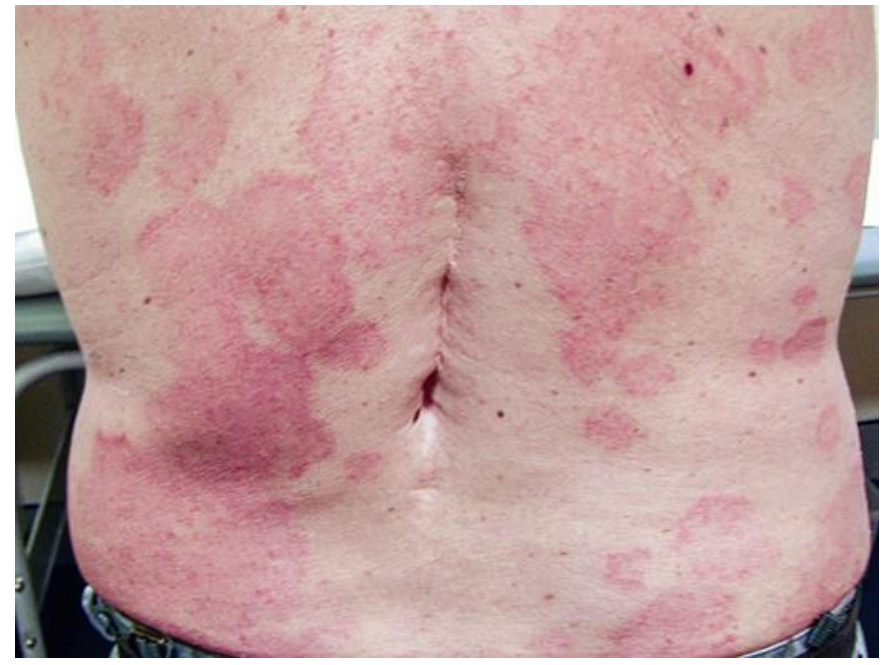

Fig. 1. Chronic plaque psoriasis on the lower back.

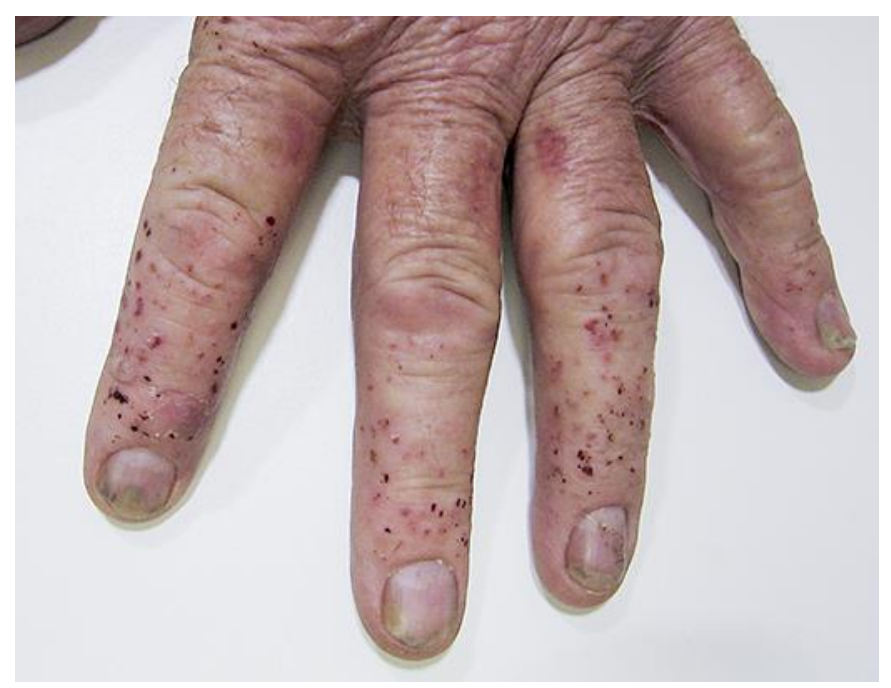

Fig. 2. Characteristic features of nail psoriasis are seen, with onycholysis, pitting and splinter hemorrhages. In addition, dermatitis herpetiformis presents atypically as vesiculobullous eruptions on the fingers. 


\section{Case Reports in Dermatology}

\begin{tabular}{l|l}
\hline Case Rep Dermatol 2021;13:141-147 \\
\hline DOI: 10.1159/000512870 & $\begin{array}{l}\text { (c) 2021 The Author(s). Published by S. Karger AG, Basel } \\
\text { www.karger.com/cde }\end{array}$ \\
\hline
\end{tabular}

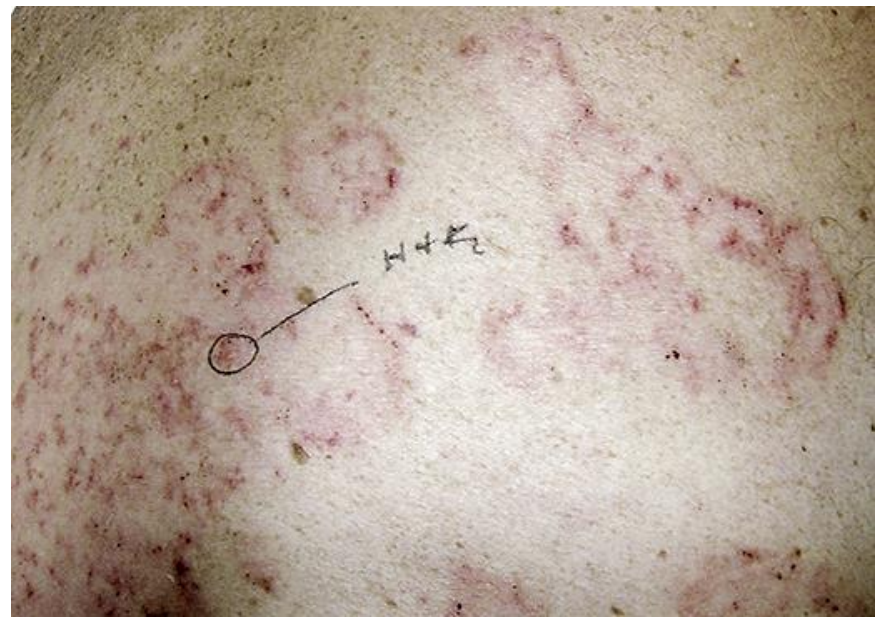

Fig. 3. Dermatitis herpetiformis presenting as multiple vesicles on the edges of psoriatic plaques located on the back. 